\title{
A search for pulsations from the compact object of GRB 060218
}

\author{
N. Mirabal ${ }^{1}$ and E. V. Gotthelf ${ }^{2}$ \\ 1 Ramón y Cajal Fellow; Dpto. de Física Atómica, Molecular y Nuclear, Universidad Complutense de Madrid, Spain \\ e-mail: mirabal@gae.ucm.es \\ 2 Columbia Astrophysics Laboratory, Columbia University, New York, NY 10027-6601, USA
}

Received 29 June 2009 / Accepted 19 December 2009

\begin{abstract}
Aims. A fraction of massive stars are expected to collapse into compact objects (accreting black holes or rapidly rotating neutron stars) that successfully produce gamma-ray bursts (GRBs). We examine the possibility of directly observing these gamma-ray burst compact objects (GCOs) using post-explosion observations of past and future GRB sites.

Methods. We present a search for early pulsations from the nearby $(z=0.0335)$ gamma-ray burst GRB 060218, which exhibited features possibly consistent with a rapidly spinning neutron star as its underlying GCO. We also consider alternative techniques that could potentially achieve a detection of GCOs either in the Local Volume or near the plane of our own Galaxy.

Results. We report the non-detection of pulsations from the GCO of GRB 060218. In particular, fast fourier transform analysis applied to the light curve shows no significant power over the range of frequencies $0.78 \mathrm{mHz}<f<227 \mathrm{~Hz}$ with an upper limit on the pulsed fraction of $\sim 2 \%$. In addition, we present detection limits of current high-resolution archival X-ray images of galaxies within the Local Volume. The existing data could be harnessed to rule out the presence of any background contaminants at the GRB position of future nearby events.

Conclusions. The null detection of pulsations from the GCO of GRB 060218 is most likely explained by the fact that the afterglow emission occurs near the head of the jet and should be far removed from the compact object. We also find that the comparison of preand post-explosion explosion images of future GRBs within the Local Volume, as well as the firm identification of a GCO within an ancient GRB remnant near the Galactic plane are extremely challenging with current $\mathrm{GeV} / \mathrm{TeV}$ capabilities. Finally, we conclude that only under some very exceptional circumstances will it be possible to directly detect the compact object responsible for gamma-ray bursts.
\end{abstract}

Key words. gamma-ray burst: individual: GBR 060218 - gamma rays: stars

\section{Introduction}

Optical spectroscopy has verified the long-hypothesized origin of gamma-ray bursts (GRBs) in the deaths of massive stars (e.g., Stanek et al. 2003; Hjorth et al. 2003). It is now accepted that "long-duration" (>2 s) GRBs accompany some core-collapse supernovae of Type Ic, in which a compact object produced during the collapse of a Wolf-Rayet progenitor powers a focused jet that bores its way through the stellar envelope (Woosley \& Bloom 2006). Unfortunately, the exact nature and eventual fate of the gamma-ray burst compact object (GCO) responsible for the the launch of the GRB jet remains unknown. The most promising alternatives include highly magnetized neutron stars (Wheeler et al. 2000; Thompson et al. 2004) and accreting black holes characterized as "collapsars" (Woosley 1993; MacFadyen \& Woosley 1999). The detection of such GCOs would not only open a new frontier in our understanding of compact object formation, but it would help us address the circumstances that give rise to a variety of stellar explosions.

Much of the effort aimed at revealing the nature of GCOs has centered on the possible interaction of the GRB with its surroundings. Brown et al. (2000) suggested that GRB explosions could lead to the formation of X-ray binaries with a black hole primary. The identification of GRB remnants in our Galaxy or in nearby galaxies has also been proposed as a possible way to pinpoint the GCO responsible for the explosion
(Perna et al. 2000; Ayal \& Piran 2001; Ramirez-Ruiz 2004). Looking for the compact object produced at the site of historical core-collapse supernovae has also been addressed by Perna et al. (2008). Using data from the Burst and Transient Experiment (BATSE) onboard the Compton Gamma Ray Observatory (CGRO), McBreen et al. (2002) inspected cumulative light curves of GRBs for possible signatures of a black hole being either spun up or down during the accretion process. Other surveys have focused on the search for gravitational waves associated with GRBs (Abbott et al. 2008). Most recently, Mazzali et al. (2006) have used spectral analysis of the broad-lined supernova SN 2006aj to infer the formation of a magnetar associated with GRB 060218. While these studies provide important insight into the origins of the explosion, further observational work is required to detect and distinguish GCOs directly.

Theoretical studies of GRBs suggest that the combination of rapid rotation and large magnetic fields in neutron stars (magnetars) could generate an energy of $10^{51}-10^{52}$ erg on a $10-100 \mathrm{~s}$ timescale required to power a long-duration GRB (Thompson et al. 2004). A direct detection of pulsations at the site of a GRB would provide strong support for such hypothesized neutron star at the center of the explosion. So far, searches for pulsation signatures have found no significant evidence of periodicity in the prompt gamma-ray (25-320 keV energy range) light curves of GRBs (Deng \& Schaefer 1997). However, very recent observations have produced tantalizing but controversial evidence of 
quasi-periodic variations in the gamma-ray light curve of GRB 090709A (Markwardt et al. 2009; Golenetskii et al. 2009; Gotz et al. 2009; Mirabal \& Gotthelf 2009).

Owing to its close proximity $(z=0.0335)$ and the early detection of an associated supernova with the Swift satellite, the observations of GRB 060218/SN 2006aj (Campana et al. 2006; Mirabal et al. 2006; Modjaz et al. 2006; Pian et al. 2006; Soderberg et al. 2006) constitute an ideal dataset to search for pulsations following the GRB onset. Additional motivation for a thorough pulsation search is provided by spectral modeling of its associated supernova SN 2006aj suggesting a nascent neutron star as the likely GCO for GRB 060218 (Mazzali et al. 2006).

In this paper, we present an early search for pulsations from the GCO of nearby GRB 060218, and an analysis of alternative observations that may produce a direct view of the GCO in the future. The paper is organized as follow. In Sect. 2, we describe a search for coherent pulsations from the GRB 060218. Section 3 discusses our results and examines potential techniques that could reveal the nature of GCOS. Finally, conclusions are presented in Sect. 4. Throughout we adopt a concordance cosmology model $\left(H_{0}=71 \mathrm{~km} \mathrm{~s}^{-1} \mathrm{Mpc}^{-1}, \Omega_{\mathrm{m}}=0.27\right.$ and $\left.\Omega_{\Lambda}=0.73\right)$.

\section{Observations and data analysis}

Timing observations of GRB 060218 were obtained with the X-ray telescope (XRT; Gehrels et al. 2004) on-board the Swift satellite. The XRT focal plane sensor is a $600 \times 602$ pixel CCD sensitive to photons in the $0.2-10 \mathrm{keV}$ energy range. Data were acquired in Windowed Timing (WT) mode starting $159 \mathrm{~s}$ after the burst. This mode provides $1.77 \mathrm{~ms}$ time resolution by rapidly clocking out the central 200 columns in the column direction with the resulting loss of imaging in one spatial dimension. We analyzed standard pipeline products created using processing version v3.9.10 made available in the Swift archive $^{1}$.

Data collected in the first orbit spanned an initial $2540 \mathrm{~s}$ with a mean count rate of 72 counts $\mathrm{s}^{-1}$ in a $1^{\prime}$ wide extraction aperture centered on the source. The light curve rises to a peak 100 counts s $^{-1}$ at the $800 \mathrm{~s}$ mark before slowly falling back to the initial detected rate of 30 counts $\mathrm{s}^{-1}$. In the next interval, following a 3160 s spacecraft orbit gap due to Earth block, the source is barely detectable (mean rate of 0.7 counts $\mathrm{s}^{-1}$ over $120 \mathrm{~s}$ ), then nearly undetectable during $22 \times 40$ s orbit-to-orbit monitoring intervals over $35 \mathrm{hrs}$, for a total of $808 \mathrm{~s}$ of exposure, resulting in 32 source counts and 8 background counts. Apart from the highly variable source flux rate, the background remained constant during all observation intervals. A light curve of the Swift events for the first interval following detection is shown is Fig. 1; note that count rates for the later observations are essential zero on this scale.

We searched for a coherent signal in the initial Swift XRT data of GRB 060218 using a fast Fourier transform (FTT). The barycenter-corrected data taken during the first orbit was rebinned in $1.77 \mathrm{~ms}$ steps and transformed using a $N=2^{21}$ element FFT. No significant power is found over the range of search frequencies $0.78 \mathrm{mHz}<f<227 \mathrm{~Hz}$ searched. The resulting power spectrum for GRB 060218 is displayed in Fig. 2 and shows characteristics of both white (power independent in frequency, mean of 2) and red noise (power modeled by a power law with best fit index $\Gamma=-2.2$ ), the latter dominating at frequencies above $30 \mathrm{mHz}$. In the white noise regime, the maximum FFT power

${ }^{1}$ See http://heasarc.gsfc.nasa.gov/docs/swift/archive/

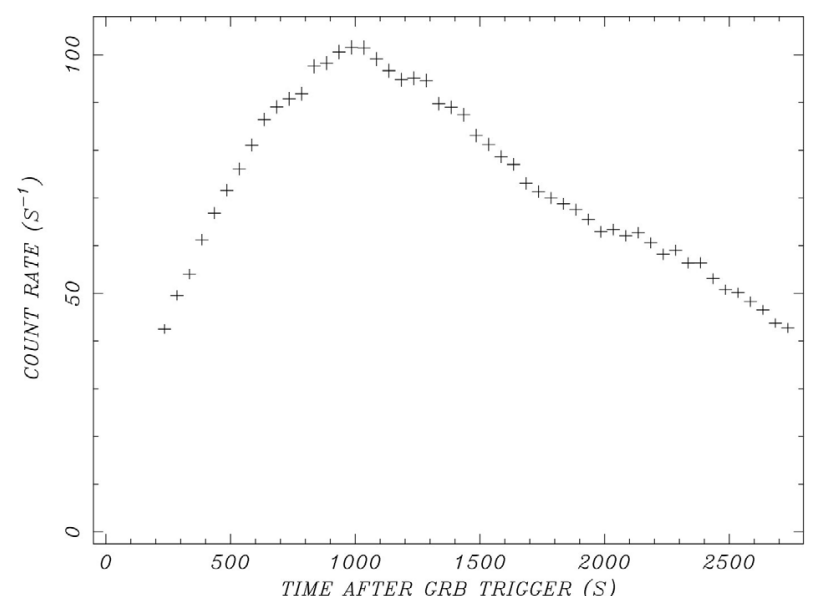

Fig. 1. Initial Swift XRT light curve of GRB 060218 acquired $159 \mathrm{~s}$ after the GRB trigger, in the $0.2-10 \mathrm{keV}$ energy band.

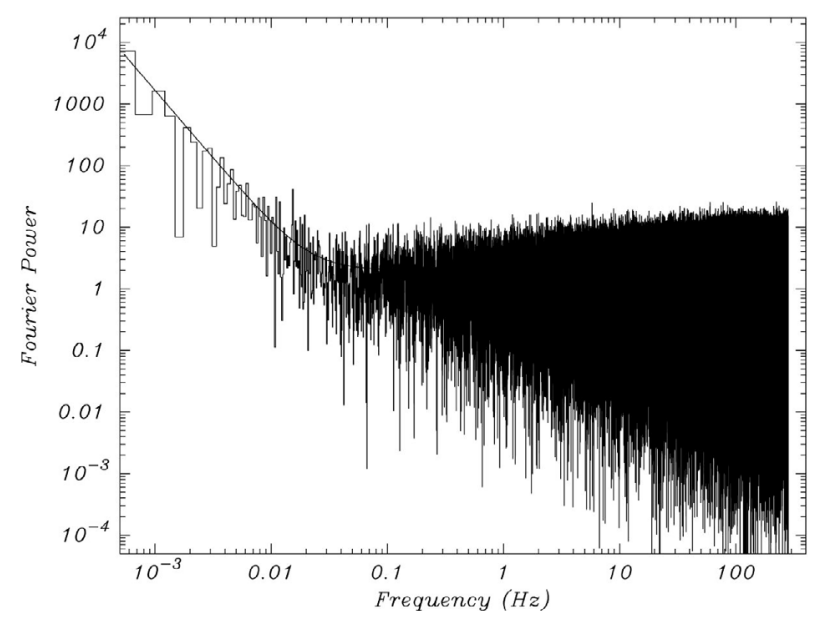

Fig. 2. Power spectrum of GRB 060218. The spectrum shows no significant coherent signal and it is dominated by red noise at low frequencies $\lesssim 0.03 \mathrm{~Hz}$.

is $S=25.67$ at $223 \mathrm{~Hz}(P=4.48 \mathrm{~ms})$. This power is consistent with random fluctuations, corresponds to a false detection probability of $\wp=N_{\text {trials }} \exp ^{-S / 2}=2^{21} \times 2.6 \times 10^{-6}>1$ for a coherence sinusoidal. Formally, the corresponding pulsed fraction limit is $f=1.8 \%(=\sqrt{2 S / N}, N=162 \mathrm{k}$ detected photons), with $50 \%$ probability. For periods in the magnetar range $(0.01 \lesssim f \lesssim 1 \mathrm{~Hz})$, which partially overlap the red noise regime, the peak FFT power is $S=37.51$ after allowing for the modeled red noise contribution, corresponding to a blind search false positive detection probability of $\wp=0.015(\approx 2.5 \sigma)$ and pulsed fraction limit of $f=2.1 \%$. Similar results are obtained using the Rayleigh, or $S=Z_{1}^{2}$ test, where the power is distributed like $\chi^{2}$ with two degrees of freedom, as for the FFT power.

To look for possible non-stationary oscillations, a similar timing analysis was done on 100-s intervals using the burst and no significant signal is found to a pulsed fraction limit of $10 \%$, (maximum FFT power $S \approx 10, N \approx 4000$ counts), with $50 \%$ probability.

\section{Discussion}

In a different astrophysical context, the absence of coherent pulsations from a newly formed compact object would be intriguing. In the case of GRBs; however, the absence of coherent 
pulsation is not totally unexpected. The reason is that the observed afterglow/breakout emission most likely occurs near the jet head far removed from the central engine (Zhang et al. 2003). As a result, temporal and spectral signatures (if any) associated with the GCO are probably swamped by the afterglow emission (Mirabal et al. 2003; Sako et al. 2005; Butler 2007).

Even in the absence of a bright afterglow, as is the case for GRB 060218, it is not entirely clear that the temporal signatures will be retained during the expansion of the jet. Consequently, we argue that early searches for pulsations in GRBs will not always provide clues about the nature of GCOs. We note, however, that our results do not necessarily rule out neutron star models for GCOs. In fact, the range of frequencies considered here may only rule out millisecond pulsars with relative slow spin periods $f<283 \mathrm{~Hz}$. As argued by Thompson et al. (2004), an energy release $E_{\gamma} \sim 10^{50}$ erg implies that the spin period of the neutron star at birth might be as low as $0.1 \mathrm{~ms}$.

Interestingly, our failed search forces us to consider alternative approaches for observing GCOs directly such as direct comparison of pre- and post-explosion X-ray images of future nearby GRB localizations (Pian et al. 2004; Kouveliotou et al. 2004) or the firm identification of a GCO within the interior of a GRB remnant in our own Galaxy (Ioka \& Mészáros 2010). In the case of pre- and post-explosion comparisons, the appearance of an X-ray source at the GRB localization, once the early GRB afterglow and underlying supernova have significantly subsided (Kouveliotou et al. 2004), would provide direct access to the GCO itself.

If GRBs originate in binary systems and the binary remains bound after the explosion (Davies et al. 2002), it is possible that a soft X-ray transient will be formed after the explosion via black hole accretion (Brown et al. 2000); or through accretion onto a neutron star from the stellar wind of an O or B companion (Lewin et al. 1995). Since GRBs are a subset of supernovae that in some cases may give birth to rapidly rotating magnetars, it makes sense to look for X-rays from an isolated rotation powered pulsar, not just an accreting binary. Perna et al. (2000) has computed the expected X-ray luminosity as a function of time for neutron stars born with different magnetic fields and spin periods. While the detection of a steady X-ray source at the site of the explosion would not by itself distinguish between a black hole or neutron star compact object, it would provide direct access to the compact object underlying the explosion.

Apart from modeling the SN and afterglow evolution, the main challenge with pre- and post-explosion comparisons is ruling out unrelated background sources such as AGN and preexisting X-ray binaries sources at the GRB site. Fortunately, there already exists an extensive high-resolution X-ray image archive of nearby galaxies acquired during the past decade (Liu 2008). The recent compilation by Liu (2008) includes 383 galaxies within $40 \mathrm{Mpc}$. After excluding early-type galaxies, this leaves a subset of 200 late-type, irregular, and peculiar galaxies within $40 \mathrm{Mpc}$. Potentially, this extensive X-ray sample can be used to to set restrictive upper limits on the X-ray emission at the GRB site prior to the explosion. Figure 3 shows the X-ray luminosity limits $L_{X}$ for 200 galaxies listed in Liu (2008) as a function of distance. Overplotted in Fig. 3 are the luminosity limits reachable for nearby GRB 980425 (Galama et al. 1998) and GRB 060218 with current X-ray telescopes assuming an $\mathrm{X}$-ray flux limit $f_{\mathrm{X}} \sim 10^{-15} \mathrm{erg} \mathrm{cm}^{-2} \mathrm{~s}^{-1}$. In the case of GRB 060218, a detection of a newly formed X-ray binary would have to exceed the levels of Eddington luminosity for a stellar-mass black hole. The situation improves for less distant events.

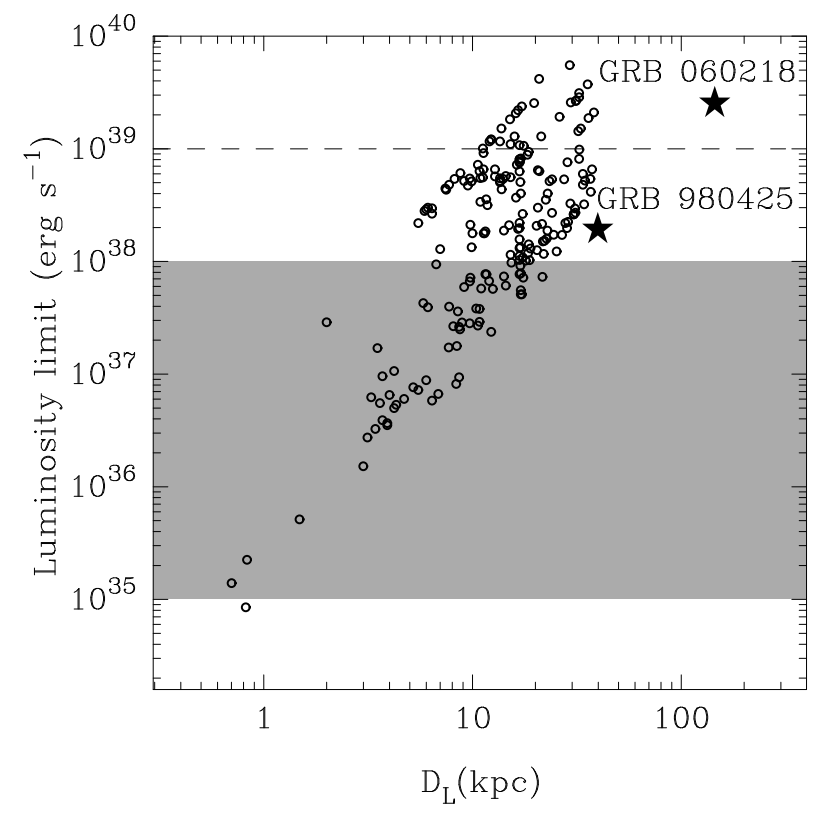

Fig. 3. X-ray luminosity limits in the $0.3-10 \mathrm{keV}$ band for the subsample of galaxies tabulated in (Liu 2008) plotted as a function of distance $D_{\mathrm{L}}$ (open circles). For comparison we also show the respective limits for GRB 980425 (Galama et al. 1998) and GRB 060218 (stars) assuming an $\mathrm{X}$-ray flux limit of $f_{\mathrm{X}}=10^{-15} \mathrm{erg} \mathrm{cm}^{-2} \mathrm{~s}^{-1}$. The shaded region shows the expected range in X-ray luminosities for X-ray binaries (Grimm et al. 2002). The dashed line near the top denotes the Eddington limit for a stellar mass black hole.

Barring the highly unlikely explosion of a GRB in our Galactic neighborhood (Stanek et al. 2006), a direct detection of a GCO embedded in a GRB remnant near the Galactic plane will have to rely heavily on studies based on morphology alone (Perna et al. 2000; Ioka \& Mészáros 2010). Numerical simulations suggest that the evolution of the GRB explosion would create non-spherical topologies that could stand out against the remnants of other stellar explosions (Ayal \& Piran 2001). However, the stability of such non-spherical morphology has been called into question given the sideways evolution of the relativistic jet (Zhang \& MacFadyen 2009). Indeed, GRB remnants may lurk within the numerous shells identified near the Galactic plane, but distinguishing them from other types of stellar remnants will be a very tall task (Daigle et al. 2007). In practical terms, the actual probability of GRB remnant detection is determined simply by the available volume that can be sampled and accurately classified. Currently, the likelihood of detecting such a GRB remnant with the available sample of high-energy sources appears limited (Aharonian et al. 2006; Holder et al. 2006; Rico 2008; Abdo et al. 2009). Fortunately, future dedicated GeV/TeV surveys could bring such detection within reach (Buckley et al. 2008).

\section{Conclusions}

In this paper we have conducted a search for early pulsations from the compact object of the nearby GRB 060218. No significant evidence of pulsations is observed. This null detection suggests that the afterglow continuum most likely swamps any pulsation/modulation signature or that the viewing angle into the progetitor is rather unfavorable. By itself, this result is not sufficient to rule out neutron star models. It should be noted that high redshifts and bright relativistic jets will challenge a sensitive search for pulsations in other GRBs detected by Swift. 
Based on our analysis, opportunities of observing GCOs directly either through their interaction with a bound stellar binary companion or via the detection of a compact object embedded within a GRB remnant near the Galactic plane offer modest hope for moving beyond afterglow science in the near future. The situation could radically change with a reasonably placed GRB in the Local Volume (e.g., SN 1987A) or through substantial progress in our $\mathrm{GeV} / \mathrm{TeV}$ capabilities. Thus, we conclude that observational progress on the nature of GCOs will once again have to rely heavily on the serendipity that is central to the origin and history of the GRB field.

Acknowledgements. We thank Jules Halpern for stimulating conversations. N.M. acknowledges support from the Spanish Ministry of Science and Technology through a Ramón y Cajal fellowship.

\section{References}

Abbott, B. P., Abbott, R., Ahikari, R., et al. 2008, Phys. Rev. D., 77, 062004

Abdo, A. A., Ackermann, M., Ajello, M., et al. 2009, ApJS, 183,46

Aharonian, F. A., Akhperjanian, A. G., Bazer-Bachi, A. R., et al. 2006, ApJ, 636, 777

Ayal, S., \& Piran, T. 2001, ApJ, 555, 23

Brown, G. E., Lee, C.-H., Wijers, R. A. M. J., et al. 2000, New Astron., 5, 191

Buckley, J., Baltz, E., Bertone, G., et al. 2008, Technical Report, [arXiv:0812.0795]

Butler, N. R. 2007, ApJ, 656, 1001

Campana, S., Mangano, V., Blustin, A. J., et al. 2006, Nature, 442, 1008

Daigle, A., Joncas, G., \& Parizeau, M. 2000, ApJ, 661, 285

Davies, M., King, A., Rosswog, S., \& Wynn, G. 2002, ApJ, 579, L63

Deng M., \& Schaefer B. E., 1997, ApJ, 491, 720

Galama, T. J., Vreeswijk, P. M., van Paradijs, J., et al. 1998, Nature, 395, 670
Gehrels, N., Chincarini, G., \& Giommi, P. 2004, ApJ, 611, 1005

Golenetskii, S., Aptekar, R., Mazets, E., et al. 2009, GCN Circular, 9647

Gotz, D., Mereghetti, S., von Kienlin, A., et al. 2009, GCN Circular, 9649

Grimm, H.-J., Gilfanov, M., \& Sunyaev, R. 2002, A\&A, 391, 923

Hjorth, J., Sollerman, J., Moller, P., et al. 2003, Nature, 423, 847

Holder, J., Atkins, R. W., Badran, H. M., et al. 2006, Astropart. Phys., 25, 391

Ioka K., \& Mészáros, P. 2010, ApJ, 709, 1337

Kouveliotou, C., Woosley, S. E., Patel, S. K., et al. 2004, ApJ, 608, 872

Lewin, W. H. G., van Paradijs, J., \& van den Heuvel, E. P. J. 1995, X-ray Binaries (Cambridge University Press), Cambridge Astrophysics Series, 26 Liu, J. 2008, [arXiv:0811.0804]

MacFadyen A. I., \& Woosley S. E., 1999, ApJ, 524, 262

Markwardt, C. B., Gavriil, F. P., Palmer, D. M., et al. 2009, GCN Circular, 9645

Mazzali, P. A., Deng, J., Nomoto, K. et al. 2006, Nature, 442, 1018

McBreen, S., McBreen, B., Hanlon, L., et al. 2002, A\&A, 393, L15

Mirabal, N., \& Gotthelf, E. V. 2009, GCN Circular, 9696

Mirabal, N., Paerels, F., \& Halpern, J. P. 2003, ApJ, 587, 128

Mirabal, N., Halpern, J. P., An, D., Thorstensen, J. R., \& Terndrup, D. M. 2006, ApJ, 643, L21

Modjaz, M., Stanek, K. Z., Garnavich, P. M., et al. 2006, ApJ, 645, L21

Perna, R., Raymond, J., \& Loeb, A. 2000, ApJ, 533, 658

Perna, R., Soria, R., Pooley, D., \& Stella, L. 2008, MNRAS, 384, 1638

Pian, E., Giommi, P., Amati, L., et al. 2004, Adv. Sp. Res., 34, 2711

Pian, E., Mazzali, P. A., Masetti, N., et al. 2006, Nature, 442, 1011

Ramirez-Ruiz, E. 2004, MNRAS, 349, L38

Rico, J. 2008, in High Energy Astronomy: Proc. of the 4th International meeting on High Energy Gamma-Ray Astronomy, ed. Aharonian F. A., Hofman W., Rieger F., AIP Conf. Proc., 1085, 183

Sako, M., Harrison, F. A., \& Rutledge, R. E. 2005, ApJ, 623, 973

Soderberg, A. M., Kulkarni, S. R., Nakar, E., et al. 2006, Nature, 442, 1014

Stanek, K. Z., Matheson, T., Garnavich P. M. et al. 2003, ApJ, 591, L17

Stanek, K. Z., Gnedin, O. Y., Beacom, J. F., et al. 2006, Acta Astron., 56, 333

Thompson, T. A., Chang, P., \& Quataert, E. 2004, ApJ, 611, 380

Wheeler, J. C., Yi, I., Höflich, P., et al. 2000, 537, 810

Woosley, S. E. 1993, ApJ, 405, 273

Woosley, S. E., \& Bloom, J. S. 2006, ARA\&A, 44, 507

Zhang, W., \& MacFadyen, A. 2009, ApJ, 698, 1261

Zhang, W., Woosley, S. E., \& MacFadyen, A. I. 2003, ApJ, 586, 356 